\title{
Profiling pro-neural to mesenchymal transition identifies a IncRNA signature in glioma
}

Qingyu Liang ${ }^{1}$, Gefei Guan ${ }^{1}$, Xue Li ${ }^{1}$, Chunmi Wei ${ }^{2}$, Jianqi Wu' ${ }^{1}$, Peng Cheng ${ }^{1}$, Anhua Wu ${ }^{1^{*}}$ and Wen Cheng ${ }^{1^{*}}$ (D)

\begin{abstract}
Background: Molecular classification has laid the framework for exploring glioma biology and treatment strategies. Pro-neural to mesenchymal transition (PMT) of glioma is known to be associated with aggressive phenotypes, unfavorable prognosis, and treatment resistance. Recent studies have highlighted that long non-coding RNAs (IncRNAs) are key mediators in cancer mesenchymal transition. However, the relationship between IncRNAs and PMT in glioma has not been systematically investigated.
\end{abstract}

Methods: Gene expression profiles from The Cancer Genome Atlas (TCGA), the Chinese Glioma Genome Atlas (CGGA), GSE16011, and Rembrandt with available clinical and genomic information were used for analyses. Bioinformatics methods such as weighted gene co-expression network analysis (WGCNA), gene set enrichment analysis (GSEA), Cox analysis, and least absolute shrinkage and selection operator (LASSO) analysis were performed.

Results: According to PMT scores, we confirmed that PMT status was positively associated with risky behaviors and poor prognosis in glioma. The 149 PMT-related IncRNAs were identified by WGCNA analysis, among which 10 (LINC01057, TP73-AS1, AP000695.4, LINC01503, CRNDE, OSMR-AS1, SNHG18, AC145343.2, RP11-25K21.6, RP1138L15.2) with significant prognostic value were further screened to construct a PMT-related IncRNA risk signature, which could divide cases into two groups with distinct prognoses. Multivariate Cox regression analyses indicated that the signature was an independent prognostic factor for high-grade glioma. High-risk cases were more likely to be classified as the mesenchymal subtype, which confers enhanced immunosuppressive status by recruiting macrophages, neutrophils, and regulatory T cells. Moreover, six IncRNAs of the signature could act as competing endogenous RNAs to promote PMT in glioblastoma.

Conclusions: We profiled PMT status in glioma and established a PMT-related 10-IncRNA signature for glioma that could independently predict glioma survival and trigger PMT, which enhanced immunosuppression.

Keywords: Glioma, PMT, IncRNA, Prognosis, ceRNA network, Immune

*Correspondence: ahwu@cmu.edu.cn; cmu071207@163.com ${ }^{1}$ Department of Neurosurgery, The First Hospital of China Medical University, Nanjing Street 155, Heping District, Shenyang 110001, Liaoning, China

Full list of author information is available at the end of the article

\section{Background}

Glioma is the most common primary brain tumor, and despite great improvements in the treatment modalities for glioma, including surgery, radiotherapy and chemotherapy, patients with glioma, especially glioblastoma multiforme (GBM), still have unfavorable outcomes $[1,2]$. To explore more effective treatments, molecular subtypes related to GBM prognosis have been identified based on large genomic data. Recently, 
GBM was classified into four molecular subtypes: pro-neural (PN), neural (NL), classical (CL), and mesenchymal (MES) [3]. Transition among the subtypes often occurs during gliomagenesis, which induces biological heterogeneity, poor prognosis, and therapeutic resistance $[4,5]$. Among the four molecular subtypes, glioma cells cultured in vitro are mostly classified into PN or MES and show distinct biological features [4]. Compared with the PN subtype, the MES subtype is associated with aggressive phenotypes and poor prognosis [6]. Additionally, remnant gliomas after radiotherapy and/or chemotherapy can undergo pro-neural to mesenchymal transition (PMT), which is associated with treatment resistance [7]. Therefore, uncovering the mechanisms that underlie PMT is urgently needed to improve glioma treatments.

Long non-coding (lnc)RNAs are non-coding RNAs of greater than $200 \mathrm{bp}$ in length. Previous studies have highlighted the important roles of lncRNAs in tumorigenesis and risky progression. Various lncRNAs have been found to be abnormally expressed in tumors, and thus are considered potential molecular targets [8]. Recently, several lncRNAs were noted to promote PMT in GBM. For example, blocking the lncRNA MIR155HG axis with the small molecule inhibitor NSC141562 suppressed mesenchymal transition [9]. Liu et al. [10] found that high LINC00152 expression might trigger PMT through the NF-KB pathway in GBM. However, a systematic understanding of how lncRNAs contribute to PMT is lacking for glioma. Hence, there is an urgent need to screen out PMT-related lncRNAs and clarify their potential mechanisms.

In this study, public glioma transcriptomic data from The Cancer Genome Atlas (TCGA), the Chinese Glioma Genome Atlas (CGGA), Rembrandt, and GSE16011 were collected for systemic analyses. We first calculated PMT scores with the single-sample gene set enrichment analysis (ssGSEA) algorithm, which reflects the PMT balance in glioma. Through this analysis, we found that PMT scores were positively associated with risky behaviors in glioma. We further identified PMT-related lncRNAs by weighted gene co-expression network analysis (WGCNA), and established a PMT-related 10-lncRNA risk signature by univariate Cox and least absolute shrinkage and selection operator (LASSO) analyses. The PMT-related IncRNA signature could independently predict the prognosis in high-grade glioma, and stimulate PMT processes to reprogram the immune microenvironment through a competing endogenous (ce)RNA network in GBM. Therefore, these findings will contribute to a deeper understanding of the mechanism of PMT, and highlight the potential application of the 10 PMT-related lncRNAs in glioma treatment strategies.

\section{Materials and methods \\ Patient datasets and clinical information}

LncRNA expression data from glioma patients was acquired from The Atlas of Noncoding RNAs in Cancer (TANRIC) [11], which was retrieved from TCGA glioma RNA-seq database (https://cancergenome.nih.gov/) and RNA-seq files from other independent studies in the CGGA database [12]. Coding mRNA expression profiles of RNAseq or microarray were collected from TCGA, CGGA, GSE16011, and Rembrandt. After acquiring the data, we annotated all samples according to their barcode ID based on available clinical information from the UCSC Xena (https://xenabrowser.net/datapages/) and CGGA (https://www.cgga.org.cn) databases. The glioma patients with detailed clinical and molecular information are described in Additional file 1: Table S1. We also downloaded matched miRNA microarray data from TCGA database.

\section{Establishing the PMT-related IncRNA signature}

To establish the PMT-related lncRNA signature, we first collected PN (M2115) and MES (M2122) gene sets from the GSEA database (https://www.gsea-msigdb.org/ gsea/index.jsp), and then calculated PN and MES scores using the ssGSEA algorithm of the GSVA package. PMT scores were generated by subtracting the PN score from the MES score. LncRNA expression profiles from TCGA RNAseq were selected as the training set. We first screened out differentially expressed lncRNAs (DELs; $|\operatorname{logFC}|>1, \quad \mathrm{P}<0.05)$ in $\mathrm{GBM}$ compared with non-GBM (oligodendroglioma, oligoastrocytoma, and astrocytoma) by the Limma package. Then the DELs were used for WGCNA analysis to identify lncRNA co-expressed modules, and to further determine PMTrelated lncRNAs with both a gene significance (GS) $>0.5$ and a module membership $(\mathrm{MM})>0.7$, as previously described [13]. PMT-related lncRNAs were used for further univariate Cox regression analyses in all glioma, non-GBM, and GBM cohorts. We identified 17 lncRNAs that significantly predicted survival in all three cohorts, and these were then used to conduct further LASSO and Cox regression analyses. The selected criteria and PMT-related lncRNA risk scores were calculated using a formula described by Chai et al. [14]. The coefficient was also used to calculate a risk score for each case in the validation dataset (CGGA). 


\section{Screening out signature-related genes (SRGs) in GBM from TCGA and CGGA}

Pearson's correlation analysis was performed to assess the relationship between coding mRNA expression and risk score in GBM. The SRGs $(\mathrm{R}>0.3, \mathrm{P}<0.05)$ were selected for Gene Ontology (GO) analysis with the ClueGO plug-in in Cytoscape [15].

\section{Constructing the PMT-related IncRNA/miRNA/mRNA network}

First, predicted binding of micro (mi)RNAs with the 10 lncRNAs were collected using the DIANA tools and LncBase Predicted v.2 (https://www.microrna.gr/LncBa se/). LncRNA/miRNA pairs and potential target genes of overlapping miRNAs were generated as described in our previous study [16]. Then, we identified miRNA-mRNA pairs by intersecting target genes with SRGs. Finally, we constructed the lncRNA/miRNA/mRNA networks with Cytoscape.

\section{Cell transfection, qRT-PCR, protein isolation and western blotting}

siRNAs for LINC01503 suppression were synthesized by GenePharma (Suzhou, China) and had the following sequences: si-LINC01503-1 sense, 5'-GGAGACAAA UGACGGCCUUTT-3'; si-LINC01503-2 sense, 5'-GGA GAAAGUUCUUUCCCUGTT-3'; and si-LINC01503-3 sense, 5'-GGACGAAUGCAGAGCCCUATT-3'. The effect of LINC01503 suppression was validated by qRT-PCR described in previous study [16]. The primer sequences were as the follows: LINC01503 (forward primer: GGGGACGGAGACAAATGAC, reverse primer: CACACTTGTCAGAGGCGTTC); CD44 (forward primer: CTGCCGCTTTGCAGGTGTA, reverse primer: CATTGTGGGCAAGGTGCTATT); OLIG2 (forward primer: TGGCTTCAAGTCATCCTCGTC, reverse primer: ATGGCGATGTTGAGGTCGTG); 18S (forward primer: GCAGAATCCACGCCAGTACAAGAT, reverse primer: TCTTCTTCAGTCGCTCCAGGTCTT). The RNA expressions of LINC01503, CD44, and OLIG2 were calculated by the $2^{-\Delta \Delta \mathrm{Ct}}$ method and normalized to $18 \mathrm{~S}$ mRNA expression. The procedure of protein isolation and western blotting was similar as that described in previous study [16]. The primary antibodies were as follows: CD44 (1:1000; Cell Signaling Technology, Boston, USA), OLIG2 (1:1000; Abcam, Cambridge, UK), $\beta$-actin (1:1000; Proteintech, Rosemont, IL, USA).

\section{Statistical analysis}

Further enrichment analysis of the biological functions between two groups was performed by GSEA (https:// software.broadinstitute.org/gsea/index.jsp). Scores of related gene sets were calculated by ssGSEA. A proteinprotein interaction (PPI) network was constructed in Cytoscape. Hub genes were generated as previously described [16]. Based on the median score, glioma cases were divided into low- and high-score groups. Differences between the two groups were estimated with the Welch $t$-test, Wilcoxon rank sum test, or $\chi^{2}$ test. Kaplan-Meier survival curves were evaluated with the log-rank test. Univariate and multivariate Cox regression analyses were performed to identify independent prognostic factors. A two-sided $\mathrm{P}$ value $<0.05$ was considered statistically significant.

\section{Results \\ PMT scores were positively associated with the risky behaviors of gliomas}

To explore the general pattern of PMT status in glioma, we compared the distribution of PMT scores according to WHO grade, histology, and molecular subtype. We found that PMT scores were increased with increased risky progression of gliomas. Cases with higher grades from TCGA RNAseq dataset exhibited higher PMT scores (Fig. 1a). According to histopathologic classification, cases with higher PMT scores were enriched in order of GBM, astrocytoma, oligoastrocytoma, and oligodendroglioma. Regarding the five molecular subtypes described in our previous study [17], PMT scores were higher in the early-GBM (LGG-IDH-wt) and GBM with wild-type IDH (GBM-IDHwt, Fig. 1a). Similar PMT score distributions were verified in TCGA microarray, CGGA RNAseq, and microarray, Rembrandt, and GSE16011 datasets (Fig. 1b, c, Additional file 2: Fig. S1A-D).

According to TCGA microarray gene expression profiles, the gliomas were classified into four subtypes [3]. The MES subtype had the riskiest progression and most unfavorable survival prognosis. Here, we found that PMT score was significantly higher in the MES subtype than in the PN subtype in multiple public glioma datasets (Fig. 1a-c, Additional file 2: Fig. S1B-D). Meanwhile, glioma cases, even GBM, with higher PMT scores had poorer survival prognoses (Fig. 1d, e, Additional file 2: Fig. S1E-M). Furthermore, there was no difference in survival between the cases with low and high PMT scores without radiotherapy or chemotherapy. However, cases with higher PMT scores that received radiotherapy or chemotherapy still suffered reduced survival time (Fig. 1f-i, Additional file 2: Fig. S2). These results suggested that the PMT balance plays a critical role in the risky progression of glioma. 


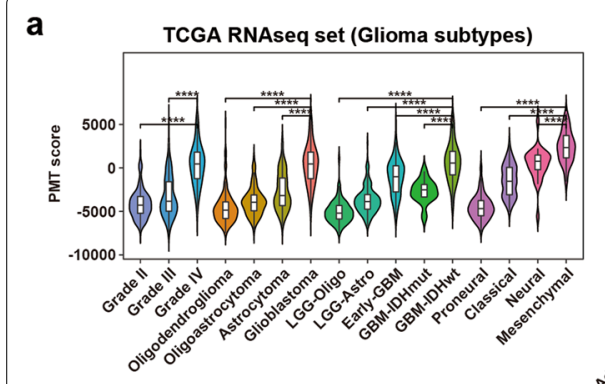

d

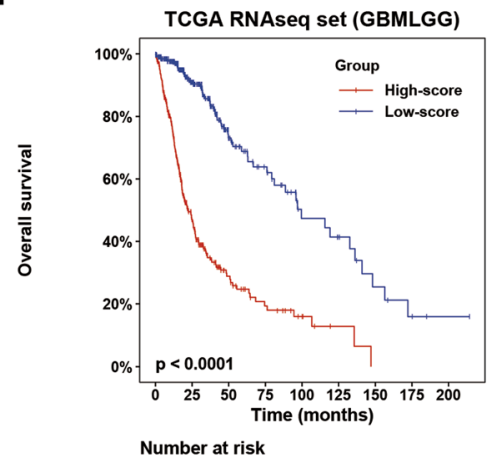

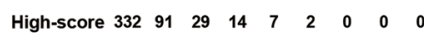
Low-score $333 \begin{array}{llllllll}162 & 56 & 35 & 16 & 12 & 6 & 3 & 1\end{array}$

e

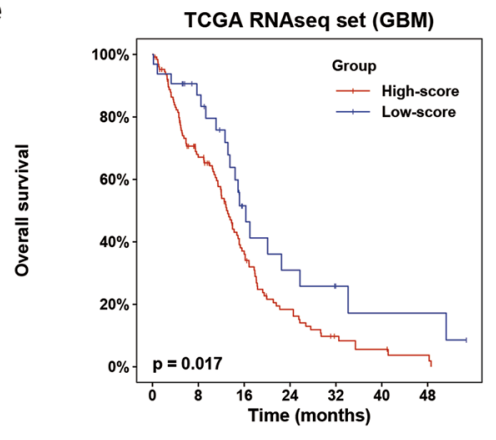

Number at risk

$\begin{array}{llllllll}\text { High-score } & 127 & 75 & 37 & 17 & 7 & 4 & 2\end{array}$

$\begin{array}{llllllll}\text { Low-score } & 32 & 24 & 10 & 6 & 3 & 2 & 2\end{array}$

g. 1 Distribution and prognostic value of PMT scores in glioma. a-c Distribution of PMT scores according to WHO grade, histopathological type, TCGA subtype, and molecular subtype in TCGA datasets and the CGGA RNAseq set. ${ }^{* * * P}<0.0001$. d, e Prognostic value of PMT scores in all glioma cases and GBM. f-i Prognostic value of PMT scores in different cohorts of GBM stratified by chemotherapy or radiotherapy in TCGA RNAseq set

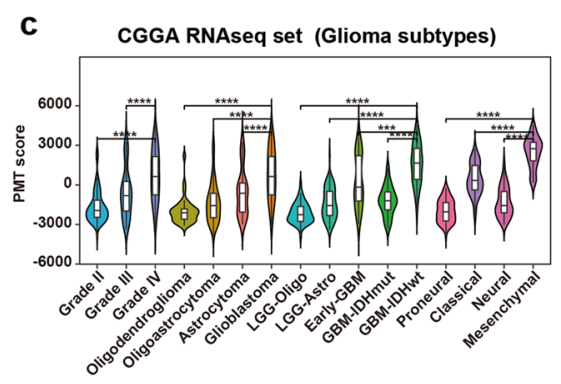

h

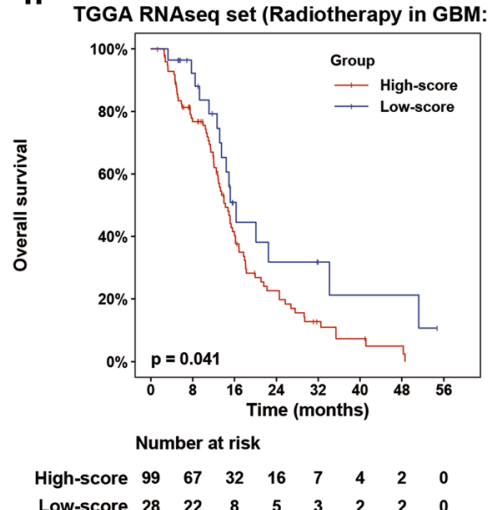

i TGGA RNAseq set (Radiotherapy in GBM: NO)

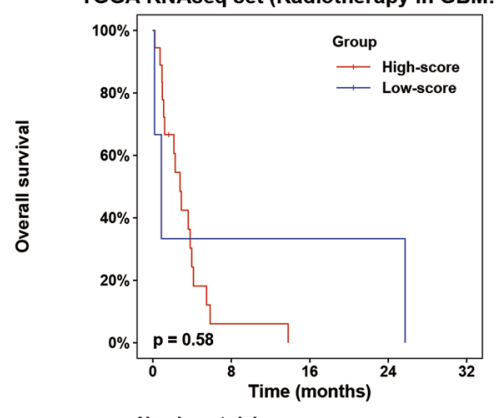

Number at risk

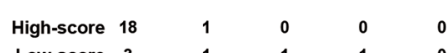

$\begin{array}{llllll}\text { High-score } & 18 & 1 & 0 & 0 & 0\end{array}$ b TCGA microarray set (Glioma subtypes)

g TGGA RNAseq set (Chemotherapy in GBM: NO)

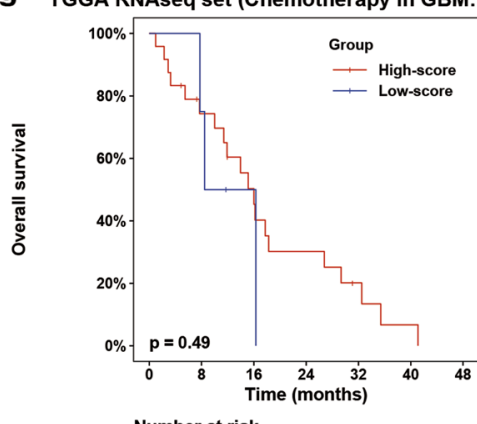

$\begin{array}{llllllll}\text { High-score } & 24 & 16 & 10 & 6 & 3 & 1 & 0\end{array}$

TGGA RNAseq set (Chemotherapy in GBM: YES)

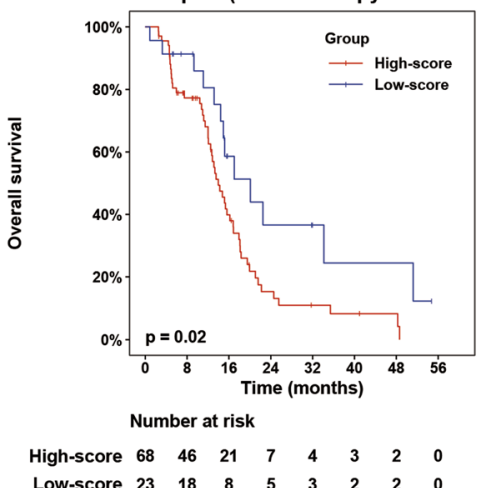

Identification of PMT related IncRNAs by WGCNA analysis To determine the PMT-related lncRNAs with potential biological functions, we conducted WGCNA analysis to generate lncRNA co-expression modules. After removing outlier cases (Additional file 2: Fig. S3A and B), 3308 DELs were divided into different modules by WGCNA cluster analysis in the training set (TCGA RNAseq set, Fig. 2a). Here, we chose $\beta=4$ as the soft threshold to build a scale-free network (Additional file 2: Fig. S3C), and then obtained 12 lncRNA co-expression modules for 


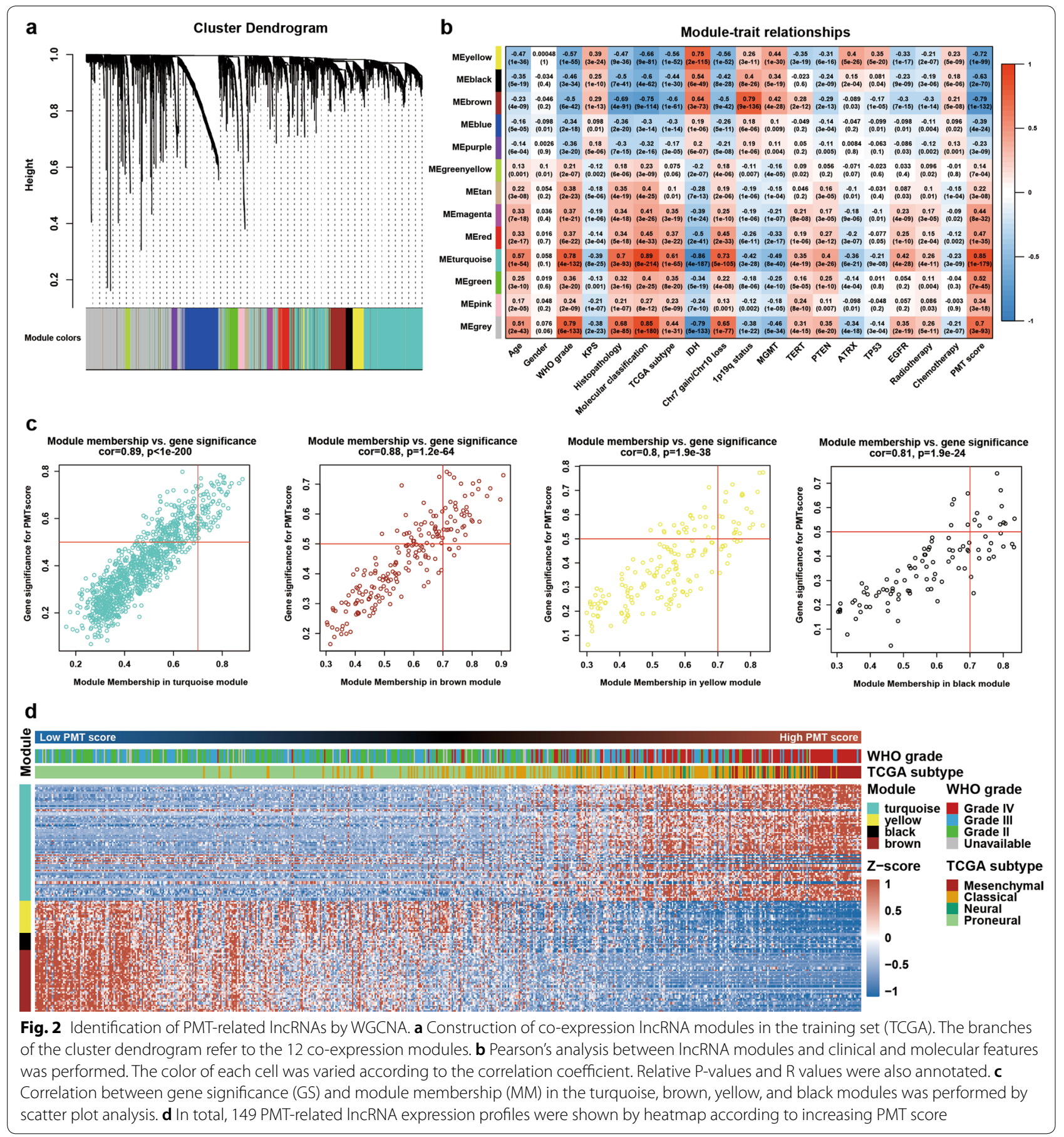

yellow, and black modules were highly negatively related to PMT scores. All four above-mentioned modules were tightly associated with high-risk clinical and molecular features, such as WHO grade, Histopathology, molecular classification, TCGA subtype, Chr 7 gain/
Chr 10 loss status, IDH status, and 1p/19q status. Thus, the turquoise, brown, yellow, and black modules were identified as modules of interest for further analyses.

Next, we screened core lncRNAs associated with PMT by setting the GS threshold to $>0.5$ and the MM threshold to $>0.7$ (Fig. 2c). This analysis identified 149 lncRNAs that were closely related to PMT (Fig. 2d 
and Additional file 1: Table S2). As expected, a strong relationship between several of these lncRNAs and mesenchymal transition in solid tumors had been reported in the literature. For example, mesenchymal transition was reinforced by CRNDE via the Wnt/ $\beta$ catenin pathway in osteosarcoma [18], and lncRNA PVT1 sponged miR-195 to enhance EMT and induce therapeutic resistance in cervical cancer [19]. Therefore, we identified 149 lncRNAs that might play important roles in regulating mesenchymal transition.

\section{Establishing a PMT-related 10-IncRNA risk signature}

To further explore the prognostic value of these PMTrelated core lncRNAs in glioma, we performed univariate Cox analyses on these lncRNAs in all glioma, non-GBM, and GBM cohorts in TCGA RNAseq set. This yielded 17 lncRNAs with significant prognostic value $(\mathrm{P}<0.05)$ in the three cohorts that were selected to generate the lncRNA risk signature (Fig. 3a, b). The lncRNAs were of two types: i.e., protective and risky; 14 lncRNAs with
$\mathrm{HR}>1$ were defined as risky, whereas three lncRNAs with $\mathrm{HR}<1$ were defined as protective.

To predict the prognosis of glioma cases by PMTrelated lncRNAs, we used TCGA RNAseq set as the training dataset to perform LASSO regression analysis on these 17 lncRNAs. Finally, 10 of the lncRNAs were selected to construct a risk signature (Fig. 3c). Meanwhile, we used coefficients obtained from LASSO analysis to calculate risk scores for each of the TCGA and CGGA glioma cases (Fig. 3d). To analyze the prognostic value of the PMT-related 10-lncRNA risk signature, glioma cases in TCGA and CGGA RNAseq sets were dichotomized based on the median risk score. There was a significant difference in survival between the low- and high-risk groups (Fig. 3e, j). Stratified analyses of TCGA and CGGA datasets showed that higher risk scores were associated with unfavorable prognosis in all WHO grades (Fig. 3f-i, k-n). In summary, we established a PMTrelated lncRNA signature with robust prognostic value in glioma.

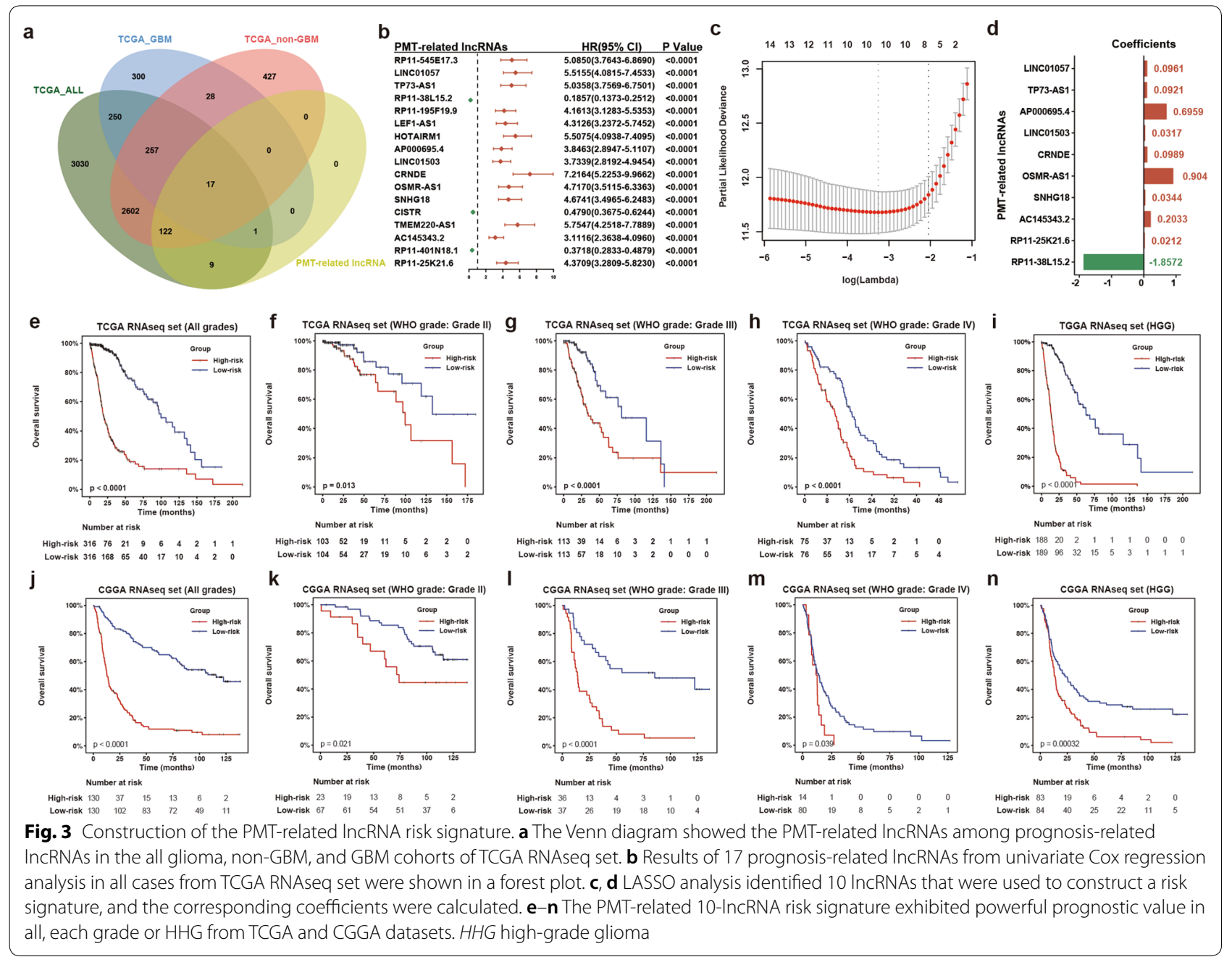




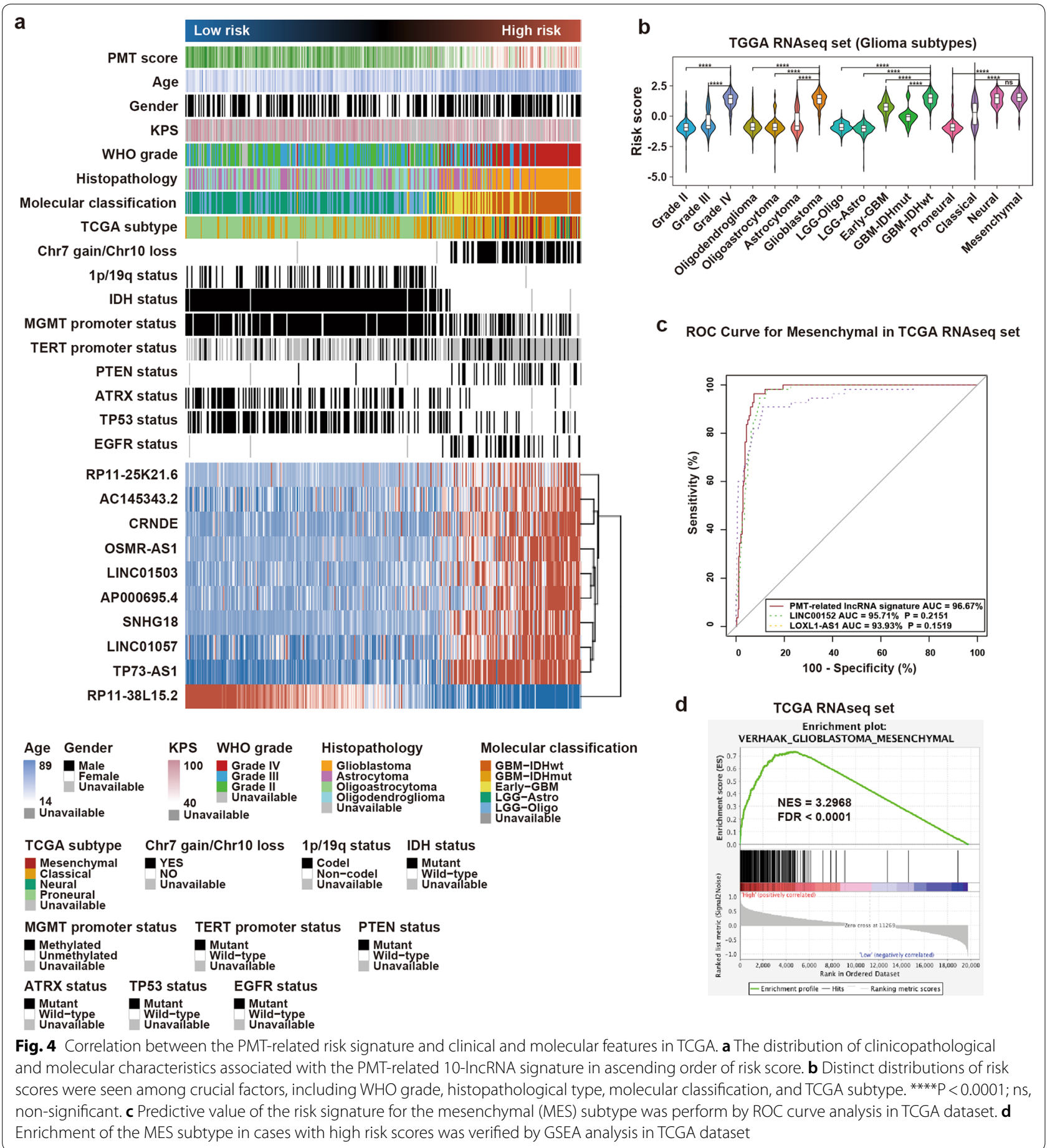

\section{The risk signature was closely related to clinical} and molecular features in glioma

The heatmaps in Fig. 4a and Additional file 2: Fig. S4A showed the distribution of clinical and molecular features according to risk score. There was a significantly different distribution in age, Karnofsky performance score
(KPS), WHO grade, histopathology, molecular subtype, TCGA subtype, Chr 7 gain/Chr 10 loss status, 1p/19q status, and related key molecular events (IDH, MGMT promoter, TERT promoter, PTEN, ATRX, TP53, and EGFR) between the high- and low-risk groups (Fig. 4a, Additional file 1: Table S3, Additional file 2: Fig. S4A). 
We also analyzed the correlation between risk score and clinical, pathological, and molecular characteristics. Cases with risky clinical factors and genomic events (MGMT promoter unmethylated, wild-type IDH, and 1p/19q non-codeletion) had higher risk scores (Fig. 4b, Additional file 2: Fig. S4B-G). Receiver operating characteristic curve (ROC) analyses indicated that risk signature might effectively predict the MES subtype in glioma. The areas under the corresponding ROC curves in TCGA and CGGA were $96.67 \%$ and $94.32 \%$, respectively, which were both more than the areas of LINC00152 and LOXL1-AS1, and which were previously reported to be associated with PMT in glioma (Fig. 4c, Additional file 2: Fig. S4H). Furthermore, GSEA confirmed that glioma cases with high risk scores were more likely to be the MES subtype, whereas cases with low risk scores were more often the PN subtype (Fig. 4d, Additional file 2: Fig. S4I and J), indicating that the lncRNA risk score could discriminate PMT status. Additionally, we found that three (TP73-AS1, LINC01503, and CRNDE) of ten PMT-related lncRNAs highly expressed both in TCGA (Additional file 2: Fig. S9A) and CGGA (Additional file 2: Fig. S9B). TP73-AS1 and CRNDE were reported to promote malignant progression of glioma [20-23]. However, researches about the impact of LINC01503 on glioma were rare. Here, we selected LINC01503 as an example to validate the impact of lncRNAs on regulating PMT in glioma cell. In TCGA and CGGA, we found the expression level of LINC01503 was positively correlated to the mesenchymal marker (CD44; Additional file 2: Fig. S9C), but negatively associated with the pro-neural marker (OLIG2; Additional file 2: Fig. S9C). In U87 glioma cell, suppression of LICN01503 could significantly inhibit the expression of CD44, but increase OLIG2 expression, suggestive of mesenchymal-to-proneural transition (Additional file 2: Fig. S9D-F). Together, these findings showed the lncRNA risk signature was positively correlated with risky behaviors in glioma.

\section{Prognostic value of the risk signature in different stratified cohorts of high-grade glioma cases}

The prognosis of high-grade (Grade III/IV) glioma cases is poor. To accurately evaluate the prognosis of these cases, we analyzed the prognostic value of the PMTrelated 10-lncRNA risk signature in different stratified cohorts of high-grade glioma cases. In TCGA, we found that the risk score had significant predictive value in cohorts stratified by age and KPS (Fig. 5a-d). There was also a significant difference in survival time between the high- and low-risk groups regardless of whether the patients received radiotherapy or chemotherapy (Fig. 5e-h).
Next, the prognostic value of the risk score in different molecular stratified cohorts was assessed. Under different statuses, such as IDH, MGMT promoter, ATRX, and TP53, cases with high risk scores had decreased survival time compared with cases with low risk scores (Fig. 5i-p). High scores indicated reduced OS in cases with wild-type EGFR and PTEN, but not in cases with mutant EGFR and PTEN (Fig. 5q-t). Similar results were validated in the CGGA dataset (Additional file 2: Fig. S5). Additionally, multivariate Cox analysis suggested that the risk signature was an independent prognostic factor in high-grade glioma (Fig. 6). In summary, the risk signature could significantly predict the prognosis of high-grade glioma.

\section{Biological functions of IncRNA in the risk signature}

To explore the different functional features of GBM cases between the high- and low-risk groups, we first screened the coding genes that were positively correlated with the risk score $(\mathrm{R}>0.3, \mathrm{p}<0.05)$ in $\mathrm{GBM}$ cases in the training dataset (TCGA, Additional file 1: Table S4). We then used the ClueGO plugin of Cytoscape to functionally annotate these coding genes. Then, we found that the most relevant biological processes in high-risk group were cell activation involved in immune response, cell adhesion and collagen-containing extracellular matrix, aminoglycan metabolic process, and positive regulation of tyrosine phosphorylation of STAT protein (Fig. 7a, b). These functional enrichments in the high-risk group were further verified by GSEA analyses (Fig. 7c). Analyses of the CGGA dataset revealed similar results (Additional file 1: Table S4; Additional file 2: Fig. S6). Additionally, previous studies have shown that STAT3 activation was positively associated with irradiation-induced PMT [24]. Taken together, we inferred that the 10 lncRNAs of the signature might facilitate biological processes that regulated PMT in GBM.

\section{GBM cases with high risk scores exhibited enhanced immunosuppression}

Recently, the MES subtype was reported to recruit more immunosuppressive cells than the $\mathrm{PN}$ and $\mathrm{CL}$ subtypes [25]. LncRNAs are important regulators of PMT, as described above; however, the correlation between PMT-related lncRNAs and immune responses in glioma are unknown. Therefore, we further analyzed immune responses related to the PMT-related lncRNA risk signature in GBM by calculating the scores of different immune cell subsets [26] by ssGSEA in the GSVA package, and immune score, stromal score, and tumor purity by the ESIMATE package [27] in GBM. Pearson's correlation analysis was performed to evaluate the relationship between these tumor microenvironment 

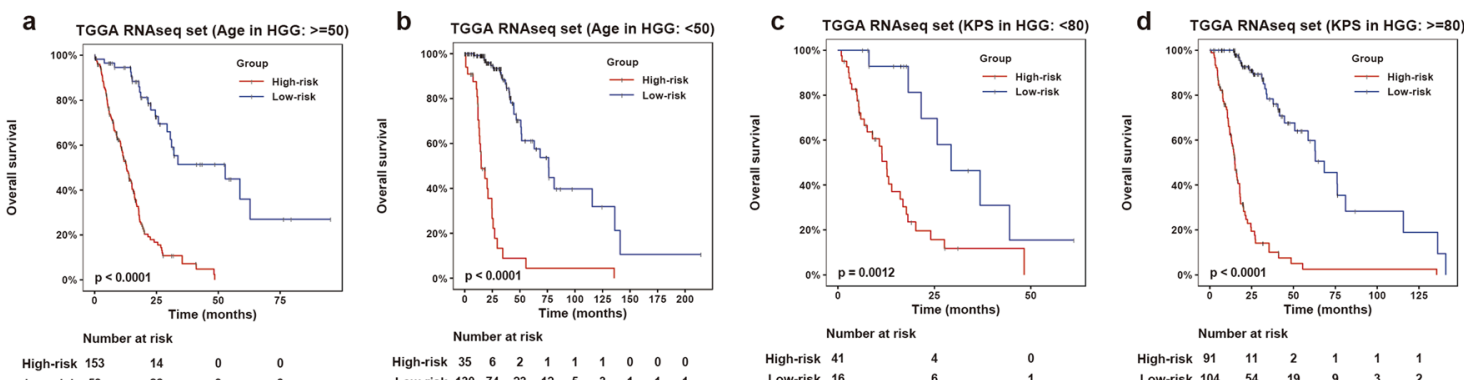

$\begin{array}{llll}\text { High-risk } & 153 & 14 & 0 \\ \text { Low-risk } & 59 & 22 & 9\end{array}$

High-risk $\begin{array}{lllllllll}35 & 6 & 2 & 1 & 1 & 1 & 0 & 0 & 0\end{array}$

$\begin{array}{ll}\text { High-risk } & 4 \\ \text { Low-risk } & 16\end{array}$

$\begin{array}{llclll}\text { High-risk } 91 & 11 & 2 & 1 & 1 & 1 \\ \text { Low-risk } 104 & 54 & 19 & 9 & 3 & 2\end{array}$

e

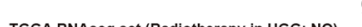

g
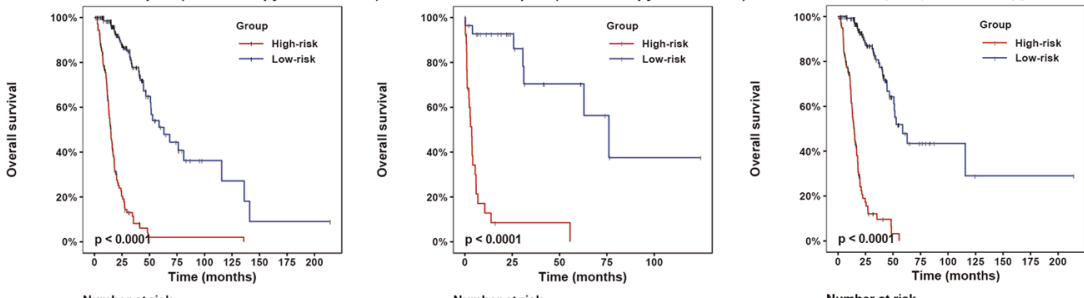

S) TGGA RNAseq set (Chemotherapy in HGG: NO) $\begin{array}{llllllllll}\text { High-risk } & 144 & 17 & 1 & 1 & 1 & 1 & 0 & 0 & 0 \\ \text { Low-risk } & 143 & 74 & 24 & 12 & 4 & 3 & 1 & 1 & 1\end{array}$ $\begin{array}{llclll}\text { High-risk } & 27 & 1 & 1 & 0 & 0 \\ \text { Low-risk } & 30 & 15 & 6 & 3 & 1\end{array}$

j

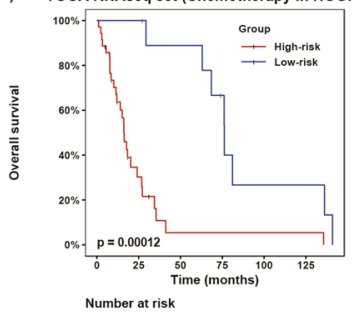
$\begin{array}{llllllllll}\text { High-risk } & 101 & 10 & 1 & 0 & 0 & 0 & 0 & 0 & 0 \\ \text { Low-risk } & 139 & 71 & 21 & 8 & 3 & 1 & 1 & 1 & 1\end{array}$ $\begin{array}{lcccccc}\text { High-risk } & 35 & 7 & 1 & 1 & 1 & 1 \\ \text { Low-risk } & 10 & 10 & 8 & 5 & 2 & 2\end{array}$ i$$
\mathbf{k}
$$$$
\text { I }
$$

TGGA RNAseq set (IDH status in HGG: Wild-type) TGGA RNAseq set (IDH status in HGG: Mutant) TGGA RNAseq set (MGMT status in HGG: Unmethylated) TGGA RNAseq set (MGMT status in HGG: Methylated)
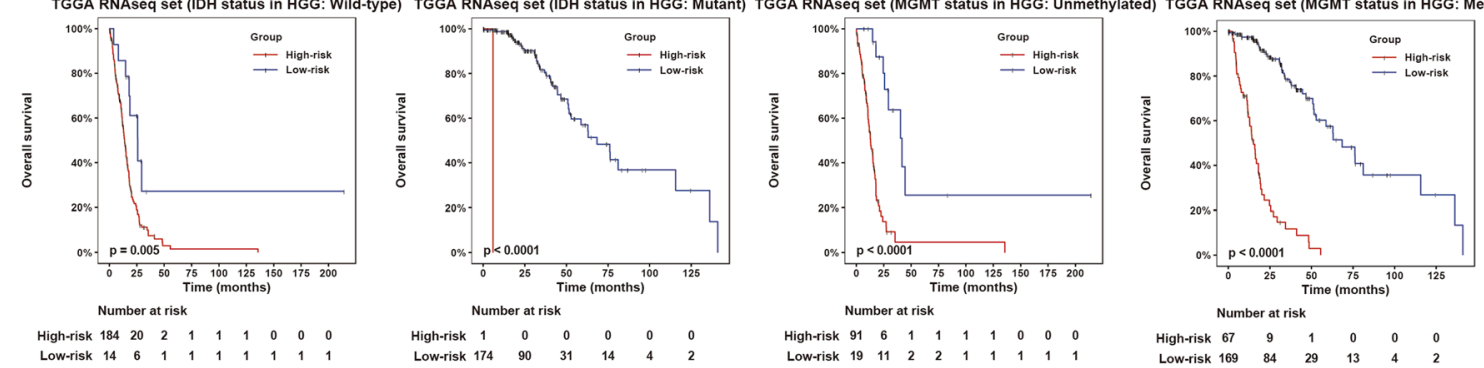

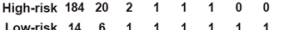

n

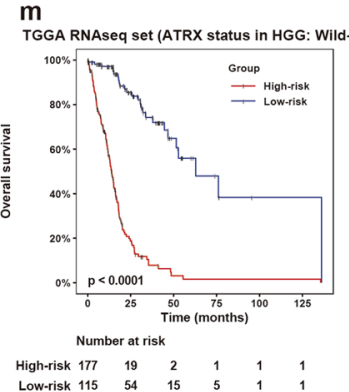

n

$\begin{array}{llllllllll}\text { High-risk } & 91 & 6 & 1 & 1 & 1 & 1 & 0 & 0 & 0 \\ \text { Low-risk } & 19 & 11 & 2 & 2 & 1 & 1 & 1 & 1 & 1\end{array}$

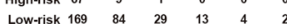

O p
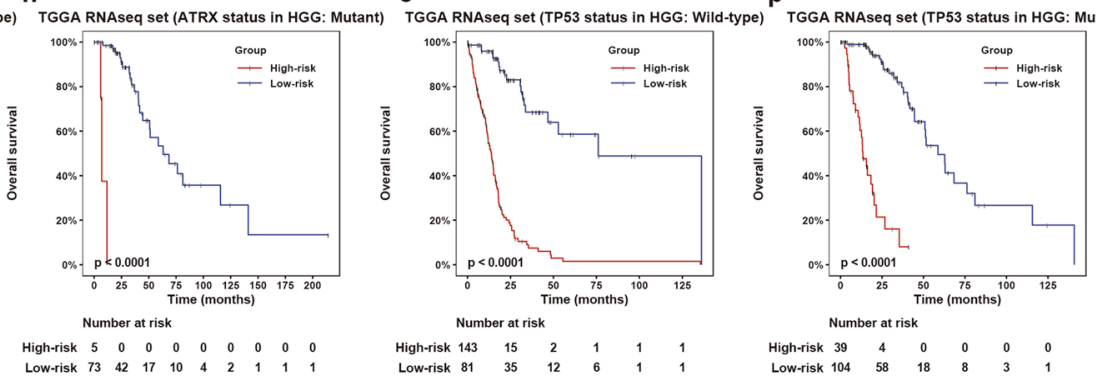

qGGA RNAseq set (EGFR status in HGG: Wild-type)

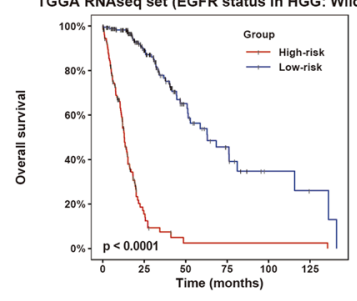

Number at risk

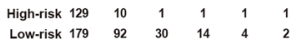

TGGA RNAseq set (EGFR status in HGG: Mutant)

S
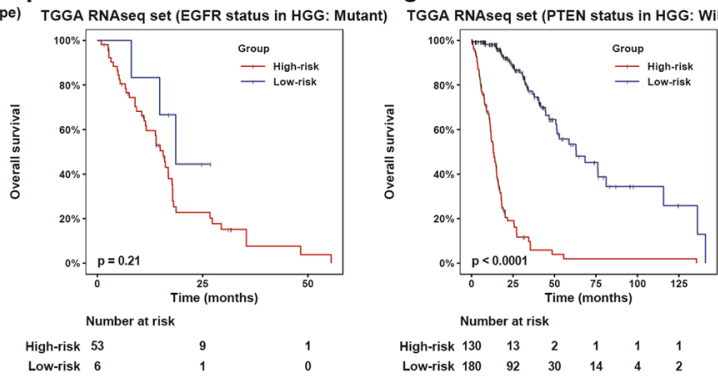

t

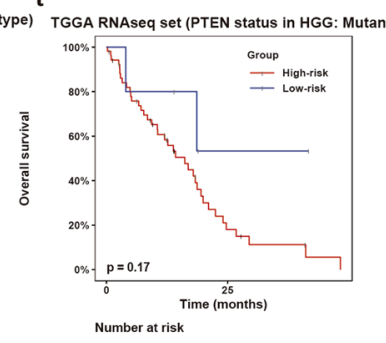

$\begin{array}{ll}\text { High-risk } & 52 \\ \text { Low-risk } & 5\end{array}$

Fig. 5 The prognostic value of the PMT-related 10-IncRNA risk signature in stratified groups of high-grade cases. $\mathbf{a}-\mathbf{h}$ The risk signature showed significant prognostic value in different cohorts stratified by clinical features in TCGA dataset. i-t Prognostic value of the risk signature was most significant in subgroups stratified by key molecular events in TCGA dataset. The p-values were computed using the log-rank test for trend 


\begin{tabular}{|c|c|c|c|c|c|c|c|}
\hline \multirow{9}{*}{ 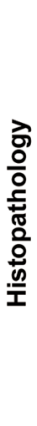 } & & & \multicolumn{2}{|c|}{ TCGA RNAseq set (HGG) } & & \multicolumn{2}{|c|}{ CGGA RNAseq set (HGG) } \\
\hline & \multicolumn{2}{|l|}{ Variables } & $\mathrm{HR}(95 \% \mathrm{Cl})$ & P Value & & $\mathrm{HR}(95 \% \mathrm{Cl})$ & P Value \\
\hline & Riskscore & $\longmapsto$ & $4.6085(1.5605-13.61)$ & 0.0057 & $\longmapsto$ & $1.8087(1.0272-3.1845)$ & 0.0401 \\
\hline & A vs GBM & 10 & $0.669(0.403-1.1107)$ & 0.1202 & $\stackrel{!}{\mapsto}$ & $0.9307(0.5742-1.5085)$ & 0.7706 \\
\hline & OA vs GBM & $\begin{array}{l}1 \\
1\end{array}$ & $0.623(0.3038-1.2776)$ & 0.1966 & $\mapsto r_{1}^{i}$ & $0.5141(0.3042-0.8688)$ & 0.013 \\
\hline & O vs GBM & 政 & $0.4127(0.2123-0.8025)$ & 0.0091 & \begin{tabular}{l|l}
1 \\
-1
\end{tabular} & $0.1499(0.0517-0.4347)$ & 0.0005 \\
\hline & Chemotherapy & 1 & $1.1232(0.7521-1.6773)$ & 0.5703 & 1 & $0.4956(0.3357-0.7316)$ & 0.0004 \\
\hline & IDH & 4 & $0.9038(0.3047-2.6807)$ & 0.8554 & $\mapsto 1$ & $0.7785(0.4438-1.3655)$ & 0.3824 \\
\hline & Age & + & $1.0273(1.0105-1.0444)$ & 0.0014 & + & $1.0003(0.9823-1.0186)$ & 0.9758 \\
\hline
\end{tabular}

Fig. 6 Multivariate Cox regression analysis of the risk signature. Forest plot showed the independent prognostic value of the risk signature compared with histopathologic classification, chemotherapy, IDH status, and age in TCGA and CGGA datasets. HGG high-grade glioma

indicators and risk score. We found that risk score was positively correlated with immune score and stromal score, but negatively correlated with tumor purity (Fig. 8a). Meanwhile, enrichment of eosinophils, macrophages, neutrophils, and regulatory $\mathrm{T}$ cells was positively associated with risk score, while central memory $\mathrm{T}$ cells and helper $\mathrm{T}$ cells were inversely related to risk score (Fig. 8b, c, Additional file 2: Fig. S7A and B). Further analyses showed that the PMT-related lncRNA risk score was positively related to the expression levels of the immunosuppressive genes described previously [28] (markers of Tregs, immunosuppressive signaling pathways, tumor-supportive macrophage chemotactic and skewing molecules, and immune suppressors; Fig. 8d, Additional file 2: Fig. S7C and D). Next, we intersected RSGs from TCGA and CGGA with immunosuppressive genes (Additional file 2: Fig. S7E). Finally, eight effectors were identified to be significantly positively correlated with the risk signature (Fig. 8e, Additional file 2: Fig. S7F). Together, these findings indicated that the ten lncRNAs could reinforce PMT to enrich certain immune cell subsets in the tumor microenvironment.

\section{Constructing the PMT-related IncRNA/miRNA/mRNA network}

Previous studies have shown that lncRNAs can act as ceRNAs to sponge miRNAs and release the coding mRNA to enhance its biological function. To explore whether any of the 10 PMT-related lncRNAs acted as ceRNAs, we predicted the potential miRNAs that could bind these lncRNAs using the DIANA website and intersected them with the downregulated miRNAs in GBM. Then, 15 miRNAs were selected for subsequent analyses. The potential target genes of these miRNAs were predicted by the starBase v3.0 database. Next, these target genes were intersected with the coding genes positively related to risk score in both TCGA and
CGGA. In total, 82 potential target genes were identified (Additional file 2: Fig. S8A).

Subsequently, we used Cytoscape to build an initial ceRNA network based on the filtered lncRNAs, miRNAs, and mRNAs (Additional file 2: Fig. S8B). Meanwhile, we constructed a protein interaction network to analyze relationships among the 82 target genes. Then, nine core genes were screened by the MCODE method, with $\mathrm{k}=2$ and degree $=3$ (Additional file 2: Fig. S8C). We further generated a core lncRNA/miRNA/hub gene network that included six lncRNAs, 10 miRNAs, and nine core coding mRNAs (Additional file 2: Fig. S8D). Further GO analyses by ClueGO showed that the functions of these nine core genes were involved in regulating leukocyte aggregation, and positive regulation of adhesion organization and regulation of apoptosis (Additional file 2: Fig. S8D). Moreover, some of the ceRNAs in the network had previously been reported to affect risky behaviors and immune responses. For example, miR-512-3p binded to CD44, resulting in suppression of invasion and cell adhesion in breast carcinoma [29]. Additionally, the mesenchymal marker CD44 was identified to significantly positively regulate PD-L1 expression in breast cancer and non-small cell lung cancer [30]. Overall, these results suggested that several PMT-related lncRNAs could promote mesenchymal transition and elicit immunosuppression via this ceRNA network in GBM.

\section{Discussion}

Verhaak et al. [3] used TCGA core gene expression profiles to classify GBM into four subtypes: PN, NL, CL, and MES. This classification system is also applicable for non-GBM. PN primarily exists in grade II/III gliomas, while CL and MES are mainly found in GBM [31]. In the study by Verhaak et al. there was no significant difference in survival time among the different subtypes [3]. However, recent studies have shown that PN cases have 


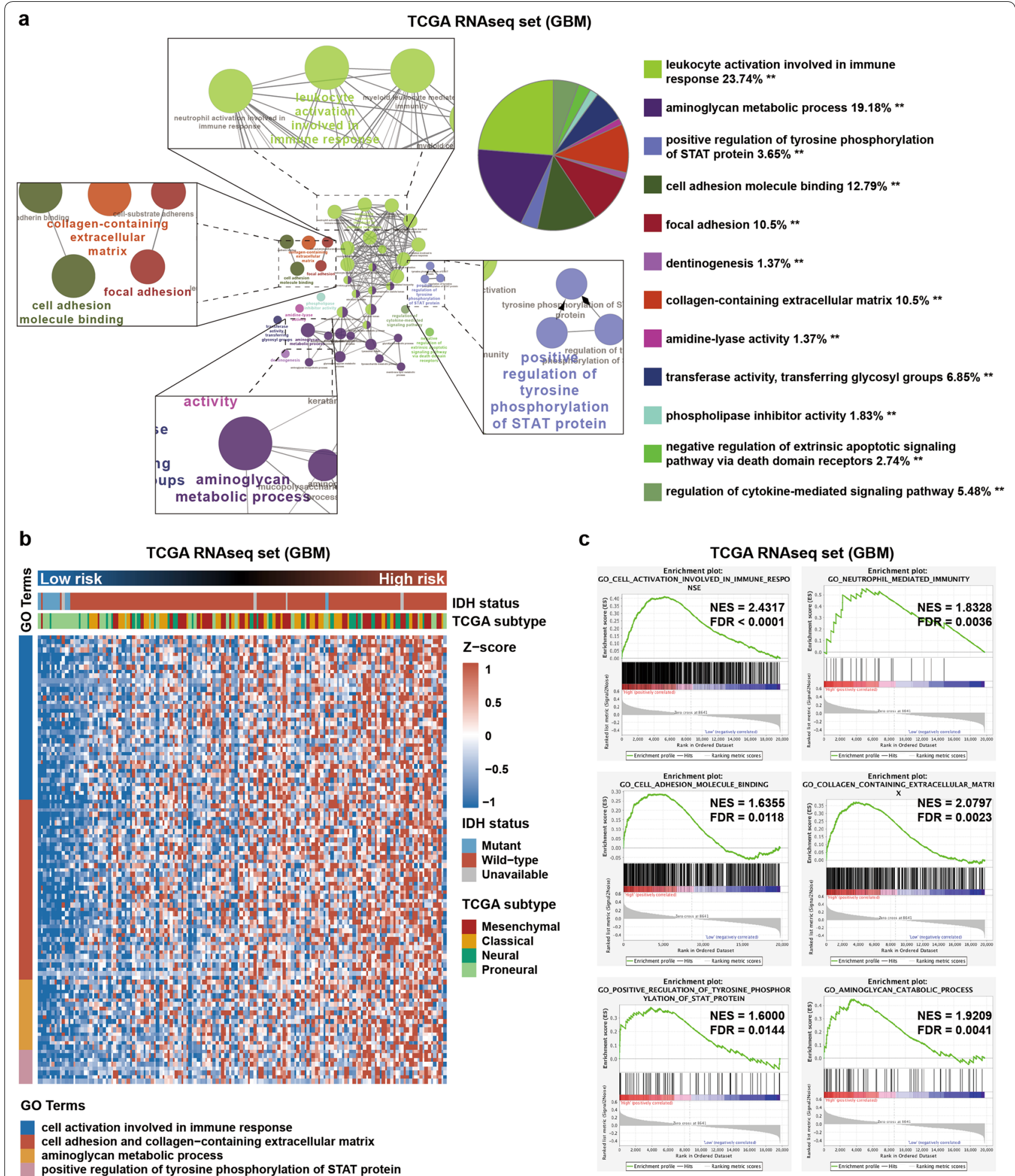

Fig. 7 Functional exploration of the risk signature in GBM. a Gene ontology $(\mathrm{GO})$ analysis using the ClueGO plugin in Cytoscape software was performed for coding genes positively correlated with risk score in TCGA. ${ }^{* *} \mathrm{P}<0.01$. b Expression profiles of genes enriched in related $\mathrm{GO}$ terms were shown in the heat map. $\mathbf{c}$ Relative biological functions of these genes were verified by GSEA analyses 


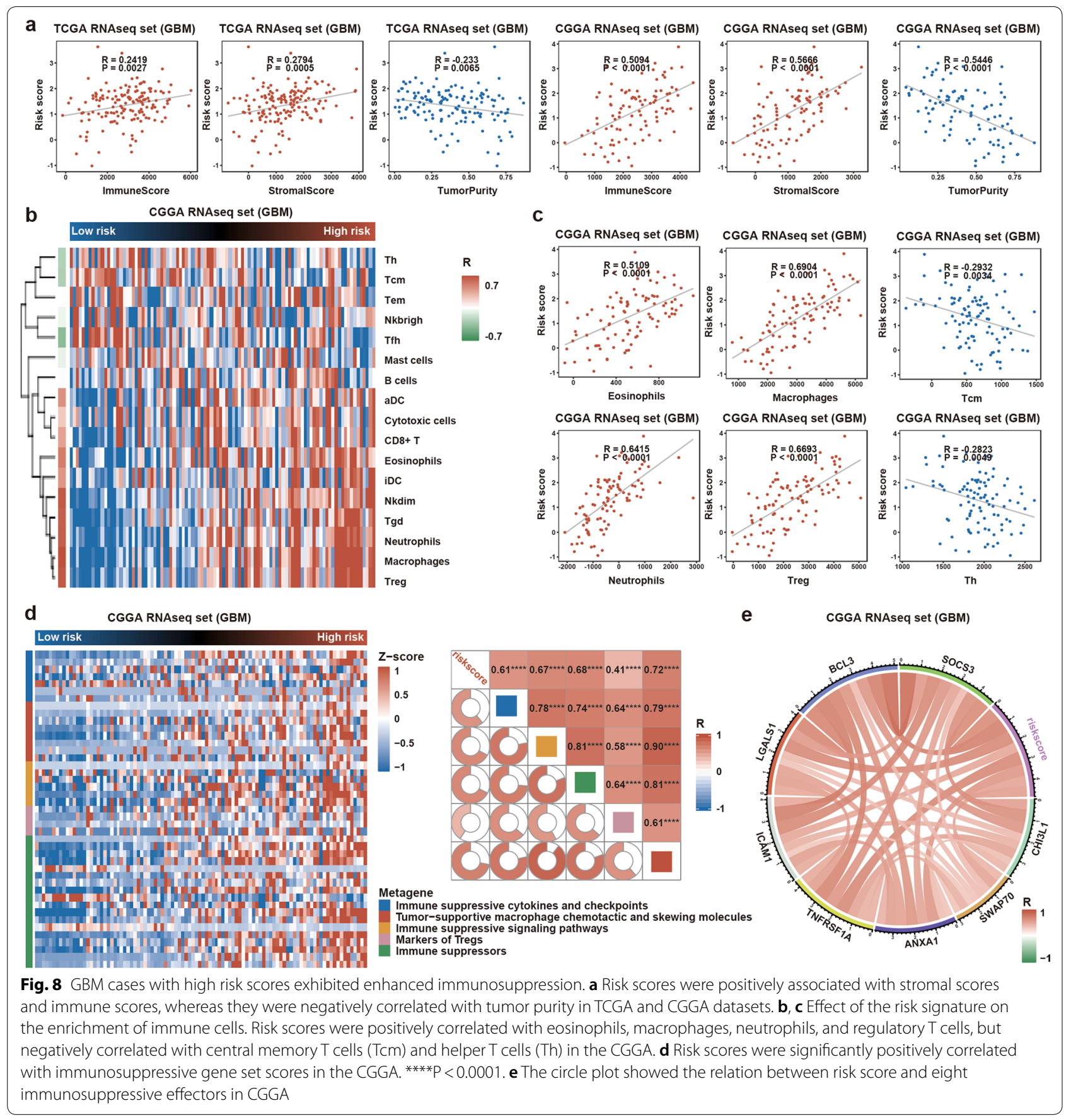

a good prognosis, NL cases have intermediate prognosis, whereas CL and MES cases display unfavorable outcomes [6]. Recently, individualized therapeutic strategies in gliomas were significantly improved by the molecular classification of glioma patients; however, the transition from PN to MES, which is induced by radiotherapy or chemotherapy, is a major trigger for treatment resistance [32]. Therefore, PMT status should be taken into account when developing an individualized treatment course. To date, there are no comprehensive methods of evaluating PMT status, meanwhile, indicators that drive PMT in glioma remain obscure. In this study, we used ssGSEA to calculate PMT scores, which could successfully estimate the PMT status in glioma. We found that PMT score was closely related to clinical and molecular characteristics in glioma. PMT scores were higher in cases with risky 
phenotypes, specifically up-regulated in IDH wild-type and MES subtype, which were recognized as subgroups with strong invasiveness and enhanced immune response $[33,34]$. Meanwhile, we also found that PMT score could predict the prognosis of glioma cases, even in GBM. Additionally, chemotherapy was more effective in cases with low PMT scores, whereas cases with high PMT scores showed less therapeutic efficacy, confirming the importance of PMT on clinical outcomes.

PMT scores were calculated from PN and MES gene sets. Although PMT score had significant prognostic value, too many effectors were included, limiting its ability for clinical translation. Recent studies have shown that lncRNAs promote mesenchymal transition in glioma. For example, H19 enhances Sox4 expression by sponging miR-130a-3p, which promotes EMT in glioma [35]. Additionally, LINC00152 regulates PMT in glioma via the miR-612/AKT2/NF-kB axis [10]. In this study, we used the WGCNA algorithm [36], which is a powerful method of identifying candidate biomarkers or therapeutic targets, to screen lncRNA co-expression modules closely related to PMT score, and identified 10 core lncRNAs associated with prognosis. The PMTrelated 10-lncRNA risk signature also had powerful predictive value. Similar to PMT scores, risk scores were positively correlated with risky behaviors and had significant predictive value for PMT status in glioma. Furthermore, a signature consisting of only 10 lncRNAs is more suitable for clinical application.

Among these 10 lncRNAs, nine (LINC01057, TP73-AS1, AP000695.4, LINC01503, CRNDE, OSMR-AS1, SNHG18, AC145343.2, and RP11-25K21.6) were high-risk lncRNAs, while only one (RP11-38L15.2) was a protective lncRNA. Several previous studies have revealed that some of these lncRNAs participate in solid tumor risky progression. For example, high TP73-AS1 expression is associated with poor prognosis, and inhibiting TP73-AS1 expression suppresses EMT in gastric cancer and bladder cancer [37, 38]. Overexpressing CRNDE promotes EMT in oral epithelial cell carcinoma and osteosarcoma [39, 40]. In glioma, high SNHG18 expression enhances radiation resistance, and triggers EMT by increasing ENO1 expression [41]. To the best of our knowledge, no studies have investigated the effects of these IncRNAs on PMT in glioma. Here, we further explored the biological functions of the PMT-related lncRNA signature. Most of the biological functions of the high-risk group were enriched in activation of immune response, regulation of cell adhesion, enhanced amino acid and glucose metabolism, and activation of STAT family genes, which are biological characteristics of the MES subtype. Moreover, previous studies have shown that inhibiting STAT3 activation suppresses radiation-induced PMT in glioma [24]. Additionally, we proved that LINC01503, one of these ten lncRNAs, could promote PMT in glioma through biological experiments. Together, these results suggest that this 10-lncRNA signature could contain potential molecular targets for glioma treatment.

The immune cells enriched in glioma play a crucial role in malignant progression and recurrence. Compared with PN, MES exhibited enhanced immunosuppression by being enriched for more tumor-associated macrophages (TAMs), CD3+, and FOXP3+ T cells [25]. Thus, the shift from PN to MES in glioma might reprogram the immunological microenvironment, resulting in enhanced tumor immunosuppression. These findings indicate that reversing PMT could improve the clinical outcomes from immunotherapy in glioma. Here, we found that the PMT-related lncRNA signature significantly reflected immune status in GBM. Risk scores were positively correlated with enrichment of eosinophils, macrophages, neutrophils, and Tregs in the glioma microenvironment. TAMs are the most abundant immune cells in glioma and create favorable conditions for growth and tumor cell invasion, resulting in unfavorable prognosis. Furthermore, we found that risk scores were positively correlated with immunosuppressive effectors, including CHI3L1, SWAP70, ANXA1, TNFRSF1A, ICAM1, LGALS1, BCL3, and SOCS3. Recent studies have shown that these immunosuppressive effectors enhance tumor immunosuppression. For example, CHI3L1 is secreted by fibroblasts and reshapes the breast cancer microenvironment by promoting the enrichment of M2 macrophages [42]. Mesenchymal stem cells can acquire immunosuppressive capacity by gaining ICAM1 expression [43]. TNFRSF1A mediates STAT3 phosphorylation and promotes the accumulation of myeloid suppressor cells in the tumor microenvironment [44]. SWAP70 restricts the maturation of dendritic cells [45]. However, very little is known about correlations between the 10 lncRNAs and eight immunosuppressive genes. Therefore, further experiments are needed to verify the mechanisms of these lncRNAs on reprogramming the immunosuppressive microenvironment in glioma.

Prior studies have noted that lncRNAs carry abundant miRNA binding sites, which could sponge miRNAs to indirectly enhance the expression of downstream target genes [46-48]. In this study, we explored whether the 10 lncRNAs in the PMT signature affected risky behaviors and immune responses via such indirect mechanisms. Finally, a core $\operatorname{lncRNA} / \mathrm{miRNA} / \mathrm{mRNA}$ network was established, which included six lncRNAs (AC145343.2, SNHG18, OSMR-AS1, AP000695.4, TP73-AS1, and LINC01057), ten miRNAs (hsa-miR103a-3p, hsa-miR-107, hsa-miR-128-3p, hsa-miR-129-5p, 
hsa-miR-338-3p, hsa-miR-381-3p, hsa-miR-410-3p, hsamiR-491-5p, hsa-miR-495-3p, and hsa-miR-512-3p), and nine core coding mRNAs (IQGAP1, CASP8, BIRC3, RAC1, MSN, ANXA5, ICAM1, CD44, and TNFRSF1A). In reviewing the literature, TP73-AS1 was found to combine with miR-124 and miR-142, resulting in enhanced proliferation of glioma cells [22, 49]. Additionally, BIRC3, ICAM1, and TNFRSF1A belong to the group of immunosuppressive factors described above, and CD44 is a core MES marker [4]. Furthermore, a previous study showed that CD44 expression was significantly inhibited by miR-512-3p in breast cancer [29]. Additionally, OPN, a physiological ligand of CD44, can bind to CD44 and activate T cells [50]. Most recently, CD44 was identified to significantly increase PD-L1 expression, enhancing immunosuppression by alleviating $\mathrm{T}$ cell enrichment in breast cancer and lung cancer [30]. According to these findings, we inferred that several PMT-related lncRNAs could indirectly promote PMT and reinforce the immunosuppressive status of GBM through a ceRNA network.

\section{Conclusions}

In summary, we explored the PMT status of glioma and identified 10 PMT-related lncRNAs in a large sample size of glioma cases. Our newly developed risk signature was constructed on the basis of these lncRNAs and could successfully evaluate PMT status. Thus, we believe that it could serve as a clinical prognostic indicator for highgrade glioma. Meanwhile, several of the identified PMTrelated lncRNAs could act as ceRNAs to trigger PMT, resulting in enhanced immunosuppression in GBM. However, the main weakness of this study was that major conclusions came from retrospective analyses of large public datasets. Further research should be undertaken to verify the biological functions and mechanism of these lncRNAs in PMT, which will provide new insights into individualized therapeutic strategies.

\section{Supplementary information}

Supplementary information accompanies this paper at https://doi. org/10.1186/s12967-020-02552-0.

Additional file 1. Table S1. Clinical and molecular information of glioma cases included in this study. Table S2. 149 PMT-related IncRNAs in four modules. Table S3. The distribution of clinical and molecular charateristics between high- and low-risk groups. Table S4. Genes positively correlated with risk score in GBM in TCGA and CGGA.

Additional file 2: Figure S1. Distribution and prognostic value of PMT scores in gliomas from multiple datasets. Figure S2. Influence of PMT score on responses to chemotherapy or radiotherapy. Figure S3. Evaluation of the distribution of glioma cases in a scale-free network. Figure S4. Associations between the PMT-related risk signature and other features in TCGA and CGGA datasets. Figure S5. The predictive value of the risk signature on survival was verified in high-grade cases from the CGGA. Figure S6. Functional enrichment of the risk signature was verified in the CGGA. Figure S7. Correlations between immunosuppressive status and the risk signature in GBM. Figure S8. Construction of the PMT-related IncRNA/miRNA/mRNA (ceRNA) network in GBM. Figure S9. The effect of LINC01503 on PMT in glioma cell.

\section{Abbreviations}

IncRNAs: Long non-coding RNAs; miRNA: MicroRNA; mRNA: Messenger RNA; TCGA: The Cancer Genome Atlas; CGGA: The Chinese Glioma Genome Atlas; GO: Gene ontology; GSEA: Gene set enrichment analysis; TANRIC: The atlas of noncoding RNAs in cancer; SRGs: Signature-related genes; logFC: Log2(fold-change); PPI: Protein-protein interaction; MCODE: Molecular complex detection; OS: Overall survival; ceRNA: Competing endogenous RNA; PN: Pro-neural; NL: Neural; CL: Classical; MES: Mesenchymal; GBM: Glioblastoma; PMT: Pro-neural to mesenchymal transition; EMT: Epithelial to mesenchymal transition; sSGSEA: Single sample gene set enrichment analysis; WGCNA: Weighted gene co-expression network analysis; WHO: World Health Organization; KPS: Karnofsky performance score; GBM-IDHwt: GBM with wildtype IDH; HR: Hazard ratio; DELs: Differentially-expressed IncRNAs.

\section{Acknowledgements}

We thank all the members of Dr. Wu AH's laboratory for help with our study.

\section{Authors' contributions}

QL, WC, and AW designed the study. QL wrote the manuscript. WC and AW revised and polished the manuscript. $Q L, G G, X L, C W, J W, P C$, and WC performed the bioinformatic and statistical analyses of the data. All authors read and approved the final manuscript.

\section{Funding}

This study was supported by National Natural Science Foundation of China (Nos. 81172409,81472360 , and 81872054 to A. Wu; No. 81872057 to P. Cheng; No. 81902546 to W. Cheng); Liaoning Science and Technology Plan Projects (no. 2011225034 to A. Wu); Natural Science Foundation of Liaoning Province (no. 20180550063 to P. Cheng); National Postdoctoral Program for Innovative Talents (no. BX20180384 to W. Cheng); Liao Ning Revitalization Talents Program (No. XLYC1807255 to W. Cheng).

\section{Availability of data and materials}

Please contact author for data requests.

\section{Ethics approval and consent to participate}

This study was approved by the Institutional Ethics Review Board of the First Hospital of China Medical University, Shenyang, China.

\section{Consent for publication}

Not applicable.

\section{Competing interests}

The authors declare that they have no competing interests.

\section{Author details}

${ }^{1}$ Department of Neurosurgery, The First Hospital of China Medical University, Nanjing Street 155, Heping District, Shenyang 110001, Liaoning, China.

${ }^{2}$ Department of Radiotherapy, The Affiliated Tumor Hospital of Guangxi Medical University, Nanning, Guangxi, China.

Received: 14 April 2020 Accepted: 27 September 2020

Published online: 07 October 2020

\section{References}

1. Ostrom QT, Bauchet L, Davis FG, Deltour I, Fisher JL, Langer CE, et al. The epidemiology of glioma in adults: a "state of the science" review. Neuro Oncol. 2014;16(7):896-913.

2. Stupp R, Hegi ME, Mason WP, van den Bent MJ, Taphoorn MJ, Janzer $\mathrm{RC}$, et al. Effects of radiotherapy with concomitant and adjuvant 
temozolomide versus radiotherapy alone on survival in glioblastoma in a randomised phase III study: 5-year analysis of the EORTC-NCIC trial. Lancet Oncol. 2009;10(5):459-66.

3. Verhaak RG, Hoadley KA, Purdom E, Wang V, Qi Y, Wilkerson MD, et al. Integrated genomic analysis identifies clinically relevant subtypes of glioblastoma characterized by abnormalities in PDGFRA, IDH1, EGFR, and NF1. Cancer Cell. 2010;17(1):98-110.

4. Bhat KPL, Balasubramaniyan V, Vaillant B, Ezhilarasan R, Hummelink $\mathrm{K}$, Hollingsworth $\mathrm{F}$, et al. Mesenchymal differentiation mediated by NF-kappaB promotes radiation resistance in glioblastoma. Cancer Cell. 2013;24(3):331-46.

5. Halliday J, Helmy K, Pattwell SS, Pitter KL, LaPlant Q, Ozawa T, et al. In vivo radiation response of proneural glioma characterized by protective p53 transcriptional program and proneural-mesenchymal shift. Proc Natl Acad Sci USA. 2014;111(14):5248-53.

6. Lin N, Yan W, Gao K, Wang Y, Zhang J, You Y. Prevalence and clinicopathologic characteristics of the molecular subtypes in malignant glioma: a multi-institutional analysis of 941 cases. PLoS ONE. 2014;9(4):e94871.

7. Fedele M, Cerchia L, Pegoraro S, Sgarra R, Manfioletti G. Proneuralmesenchymal transition: phenotypic plasticity to acquire multitherapy resistance in glioblastoma. Int J Mol Sci. 2019;20(11):2746.

8. Arun G, Diermeier SD, Spector DL. Therapeutic targeting of long non-coding RNAs in cancer. Trends Mol Med. 2018:24(3):257-77.

9. Wu X, Wang Y, Yu T, Nie E, Hu Q, Wu W, et al. Blocking MIR155HG/ miR-155 axis inhibits mesenchymal transition in glioma. Neuro Oncol. 2017:19(9):1195-205.

10. Cai J, Zhang J, Wu P, Yang W, Ye Q, Chen Q, et al. Blocking LINC00152 suppresses glioblastoma malignancy by impairing mesenchymal phenotype through the miR-612/AKT2/NF-kappaB pathway. J Neurooncol. 2018;140(2):225-36.

11. Li J, Han L, Roebuck P, Diao L, Liu L, Yuan Y, et al. TANRIC: an interactive open platform to explore the function of IncRNAs in cancer. Cancer Res. 2015;75(18):3728-37.

12. Bao ZS, Chen HM, Yang MY, Zhang CB, Yu K, YeWL, et al. RNA-seq of 272 gliomas revealed a novel, recurrent PTPRZ1-MET fusion transcript in secondary glioblastomas. Genome Res. 2014;24(11):1765-73.

13. Pan $\mathrm{S}$, Zhan Y, Chen X, Wu B, Liu B. Identification of biomarkers for controlling cancer stem cell characteristics in bladder cancer by network analysis of transcriptome data stemness indices. Front Oncol. 2019:9:613.

14. Chai RC, Wu F, Wang QX, Zhang S, Zhang KN, Liu YQ, et al. m(6) A RNA methylation regulators contribute to malignant progression and have clinical prognostic impact in gliomas. Aging (Albany NY). 2019;11(4):1204-25.

15. Shannon P, Markiel A, Ozier O, Baliga NS, Wang JT, Ramage D, et al. Cytoscape: a software environment for integrated models of biomolecular interaction networks. Genome Res. 2003;13(11):2498-504.

16. Liang $\mathrm{Q}, \mathrm{Li}$ X, Guan $\mathrm{G}, \mathrm{Xu}$ X, Chen C, Cheng P, et al. Long non-coding RNA, HOTAIRM1, promotes glioma malignancy by forming a ceRNA network. Aging (Albany NY). 2019;11(17):6805-38.

17. Zhang C, Cheng W, Ren X, Wang Z, Liu X, Li G, et al. Tumor purity as an underlying key factor in glioma. Clin Cancer Res. 2017;23(20):6279-91.

18. Ding Q, Mo F, Cai X, Zhang W, Wang J, Yang S, et al. LncRNA CRNDE is activated by SP1 and promotes osteosarcoma proliferation, invasion, and epithelial-mesenchymal transition via Wnt/beta-catenin signaling pathway. J Cell Biochem. 2020;121:3358-71.

19. Shen CJ, Cheng YM, Wang CL. LncRNA PVT1 epigenetically silences miR-195 and modulates EMT and chemoresistance in cervical cancer cells. J Drug Target. 2017;25(7):637-44.

20. Wang Y, Wang Y, Li J, Zhang Y, Yin H, Han B. CRNDE, a long-noncoding RNA, promotes glioma cell growth and invasion through mTOR signaling. Cancer Lett. 2015;367(2):122-8.

21. Zheng J, Liu X, Wang P, Xue Y, Ma J, Qu C, et al. CRNDE promotes malignant progression of glioma by attenuating miR-384/PIWIL4/STAT3 axis. Mol Ther. 2016;24(7):1199-215.

22. Zhang $R$, Jin H, Lou F. The long non-coding RNA TP73-AS1 interacted with miR-142 to modulate brain glioma growth through HMGB1/RAGE pathway. J Cell Biochem. 2018;119(4):3007-166.

23. Mazor G, Levin L, Picard D, Ahmadov U, Caren H, Borkhardt A, et al. The IncRNA TP73-AS1 is linked to aggressiveness in glioblastoma and promotes temozolomide resistance in glioblastoma cancer stem cells. Cell Death Dis. 2019;10(3):246.

24. Lau J, Ilkhanizadeh S, Wang S, Miroshnikova YA, Salvatierra NA, Wong RA, et al. STAT3 blockade inhibits radiation-induced malignant progression in glioma. Cancer Res. 2015;75(20):4302-11.

25. Kaffes I, Szulzewsky F, Chen Z, Herting CJ, Gabanic B, Velazquez Vega JE, et al. Human mesenchymal glioblastomas are characterized by an increased immune cell presence compared to proneural and classical tumors. Oncoimmunology. 2019:8(11):e1655360.

26. Tamborero D, Rubio-Perez C, Muinos F, Sabarinathan R, Piulats JM, Muntasell A, et al. A pan-cancer landscape of interactions between solid tumors and infiltrating immune cell populations. Clin Cancer Res. 2018;24(15):3717-28.

27. Yoshihara K, Shahmoradgoli M, Martinez E, Vegesna R, Kim H, Torres-Garcia $W$, et al. Inferring tumour purity and stromal and immune cell admixture from expression data. Nat Commun. 2013;4:2612.

28. Doucette T, Rao G, Rao A, Shen L, Aldape K, Wei J, et al. Immune heterogeneity of glioblastoma subtypes: extrapolation from the cancer genome atlas. Cancer Immunol Res. 2013;1 (2):112-22.

29. Rutnam ZJ, Yang BB. The non-coding 3'UTR of CD44 induces metastasis by regulating extracellular matrix functions. J Cell Sci. 2012;125(Pt 8):2075-85.

30. Kong T, Ahn R, Yang K, Zhu X, Fu Z, Morin G, et al. CD44 promotes PD-L1 expression and its tumor-intrinsic function in breast and lung cancers. Cancer Res. 2020;80(3):444-57.

31. Guan $X$, Vengoechea J, Zheng S, Sloan AE, Chen Y, Brat DJ, et al. Molecular subtypes of glioblastoma are relevant to lower grade glioma. PLOS ONE. 2014;9(3):e91216.

32. Segerman A, Niklasson M, Haglund C, Bergstrom T, Jarvius M, Xie Y, et al. Clonal variation in drug and radiation response among gliomainitiating cells is linked to proneural-mesenchymal transition. Cell Rep. 2016;17(11):2994-3009.

33. Amankulor NM, Kim Y, Arora S, Kargl J, Szulzewsky F, Hanke M, et al. Mutant $\mathrm{IDH} 1$ regulates the tumor-associated immune system in gliomas. Genes Dev. 2017;31(8):774-86.

34. Carro MS, Lim WK, Alvarez MJ, Bollo RJ, Zhao X, Snyder EY, et al. The transcriptional network for mesenchymal transformation of brain tumours. Nature. 2010;463(7279):318-25.

35. Hu Q, Yin J, Zeng A, Jin X, Zhang Z, Yan W, et al. H19 functions as a competing endogenous RNA to regulate EMT by sponging miR-130a-3p in glioma. Cell Physiol Biochem. 2018;50(1):233-45.

36. Langfelder P, Horvath S. WGCNA: an R package for weighted correlation network analysis. BMC Bioinform. 2008;9:559.

37. Tuo Z, Zhang J, Xue W. LncRNA TP73-AS1 predicts the prognosis of bladder cancer patients and functions as a suppressor for bladder cancer by EMT pathway. Biochem Biophys Res Commun. 2018;499(4):875-81.

38. Zhang W, Zhai Y, Wang W, Cao M, Ma C. Enhanced expression of IncRNA TP73-AS1 predicts unfavorable prognosis for gastric cancer and promotes cell migration and invasion by induction of EMT. Gene. 2018;678:377-83.

39. Li Z, Tang Y, Xing W, Dong W, Wang Z. LnCRNA, CRNDE promotes osteosarcoma cell proliferation, invasion and migration by regulating Notch1 signaling and epithelial-mesenchymal transition. Exp Mol Pathol. 2018;104(1):19-25.

40. Dai J, Mu JW, Mu H. Long non-coding RNA CRNDE regulates cell proliferation, migration, invasion, epithelial-mesenchymal transition and apoptosis in oral squamous cell carcinoma. Oncol Lett. 2019;17(3):3330-40.

41. Zheng $R$, Yao $Q, L i X, X u$ B. Long noncoding ribonucleic acid SNHG18 promotes glioma cell motility via disruption of alpha-enolase nucleocytoplasmic transport. Front Genet. 2019;10:1140.

42. Cohen N, Shani O, Raz Y, Sharon Y, Hoffman D, Abramovitz L, et al. Fibroblasts drive an immunosuppressive and growth-promoting microenvironment in breast cancer via secretion of Chitinase 3-like 1. Oncogene. 2017;36(31):4457-68.

43. Ren $\mathrm{G}$, Zhao X, Zhang L, Zhang J, L'Huillier A, Ling W, et al. Inflammatory cytokine-induced intercellular adhesion molecule-1 and vascular cell adhesion molecule-1 in mesenchymal stem cells are critical for immunosuppression. J Immunol. 2010;184(5):2321-8.

44. Sobo-Vujanovic A, Vujanovic L, DeLeo AB, Concha-Benavente F, Ferris RL, Lin Y, et al. Inhibition of soluble tumor necrosis factor prevents chemically induced carcinogenesis in mice. Cancer Immunol Res. 2016;4(5):441-51.

45. Ocana-Morgner C, Gotz A, Wahren C, Jessberger R. SWAP-70 restricts spontaneous maturation of dendritic cells. J Immunol. 2013;190(11):5545-58. 
46. Cui Y, Yi L, Zhao JZ, Jiang YG. Long noncoding RNA HOXA11-AS functions as miRNA sponge to promote the glioma tumorigenesis through targeting miR-140-5p. DNA Cell Biol. 2017;36(10):822-8.

47. Shang C, Guo Y, Hong Y, Xue YX. Long non-coding RNA TUSC7, a target of miR-23b, plays tumor-suppressing roles in human gliomas. Front Cell Neurosci. 2016;10:235.

48. Jia P, Cai H, Liu X, Chen J, Ma J, Wang P, et al. Long non-coding RNA H19 regulates glioma angiogenesis and the biological behavior of gliomaassociated endothelial cells by inhibiting microRNA-29a. Cancer Lett. 2016;381(2):359-69.

49. Xiao S, Wang R, Wu X, Liu W, Ma S. The Long noncoding RNATP73-AS1 interacted with miR-124 to modulate glioma growth by targeting inhibitor of apoptosis-stimulating protein of p53. DNA Cell Biol. 2018;37(2):117-25.
50. Klement JD, Paschall AV, Redd PS, Ibrahim ML, Lu C, Yang D, et al. An osteopontin/CD44 immune checkpoint controls CD8+T cell activation and tumor immune evasion. J Clin Invest. 2018:128(12):5549-60.

\section{Publisher's Note}

Springer Nature remains neutral with regard to jurisdictional claims in published maps and institutional affiliations.
Ready to submit your research? Choose BMC and benefit from:

- fast, convenient online submission

- thorough peer review by experienced researchers in your field

- rapid publication on acceptance

- support for research data, including large and complex data types

- gold Open Access which fosters wider collaboration and increased citations

- maximum visibility for your research: over $100 \mathrm{M}$ website views per year

At BMC, research is always in progress.

Learn more biomedcentral.com/submissions 